\title{
. THE STRUCTURED FLOW ON THE BRAIN'S RESTING STATE MANIFOLD
}

${ }_{5}$ Jan Fousek $^{1^{*}}$, Giovanni Rabuffo ${ }^{1}$, Kashyap Gudibanda ${ }^{1}$, Hiba Sheheitli ${ }^{1}$, Viktor Jirsa $^{1 *}$, - Spase Petkoski ${ }^{\text {* }}$

$7{ }^{1}$ Aix Marseille University, INSERM, INS, Institut de Neurosciences des Systèmes, 13005 Marseille, \& France

\begin{abstract}
Spontaneously fluctuating brain activity patterns emerge at rest and relate to brain functional networks involved in task conditions. Despite detailed descriptions of the spatio-temporal brain patterns, our understanding of their generative mechanism is still incomplete. Using a combination of computational modeling and dynamical systems analysis we provide a complete mechanistic description in terms of the constituent entities and the productive relation of their causal activities leading to the formation of a resting state manifold via the network connectivity. We demonstrate that the symmetry breaking by the connectivity creates a characteristic flow on the manifold, which produces the major empirical data features including spontaneous high amplitude co-activations, neuronal cascades, spectral cortical gradients, multistability and characteristic functional connectivity dynamics. The understanding of the brain's resting state manifold is fundamental for the construction of task-specific flows and manifolds used in theories of brain function such as predictive coding.
\end{abstract}

\section{Introduction}

The human brain at rest exhibits remarkable richness of neural activity structured both in time and space. Early computational modeling studies explored how these spontaneous fluctuations are constrained and how their organisation is shaped by the anatomic connectivity (Honey et al., 2007; Deco et al., 2013; Cabral et al., 2017) enabling to start disentangling the mechanisms of the resting state dynamics in silico. A substantial body of work has related the emergent activity patterns at rest to the brain functional networks involved in task conditions (Gusnard et al., 2001; Damoiseaux et al., 2006), and shown that the spatio-temporal variability of resting-state activity possesses functional significance (Deco et al., 2011; Hutchison et al., 2013; Preti et al., 2017), relevance to cognitive task performance (Shine et al., 2016), consciousness levels (Cavanna et al., 2018), changes during ageing (Battaglia et al., 2020), mental disorders (Braun et al., 2018), and neurodegenerative diseases (e.g. Alzheimer's dementia; Jones et al. (2012)). The structure of the resting state dynamics changes over time (Zalesky et al., 2014) and is characterized by a range of properties such

*For correspondence: jan.fousek@univ-amu.fr (JF); viktor.jirsa@univ-amu.fr (VJ) 
as metastability (Baker et al., 2014; beim Graben et al., 2019), event-like coactivations (Tagliazucchi et al., 2012; Liu et al., 2018; Esfahlani et al., 2020) and traveling waves (Gu et al., 2021). However, our understanding of the mechanisms underlying these spatio-temporal patterns of the brain activity at rest is still incomplete (Mišic et al., 2016) and whole brain network models have a crucial role to play on that front (Breakspear, 2017).

There is general agreement that the resting brain operates near criticality. This is supported by a large range of analyses performed on simulated and empirical data using network based measures (functional connectivity, functional connectivity dynamics), information theoretical measures (entropy, ignition) and descriptions of spatiotemporal dynamics (avalanches, cascades). Modeling efforts provide further evidence for the close relationship between the empirical data features and the properties of the structural network, local dynamics, coupling strength, neural gain (Deco et al., 2009, 2021; Melozzi et al., 2019; Shine et al., 2018; Cabral et al., 2017; Roberts et al., 2019; Courtiol et al., 2020). The resting state dynamics can then be understood as noise-driven fluctuations of brain activity, operating near criticality and constrained by the brain connectivity (Ghosh et al., 2008; Hansen et al., 2015). However, none of the above qualifies as a description of a mechanism. Descriptions of mechanisms require formulation in terms of causal activities of their constituent entities and render the end stage, in our context the resting state dynamics, intelligible by showing how it is produced (Machamer et al., 2000). To explain is thus not merely to redescribe one regularity (e.g. functional connectivity dynamics, or maximization of entropy) as a series of several (such as near-criticality, cascades, ignition). Rather, explanation involves revealing the productive relation between causal activities linked to their constituent entities.

In this paper we aim to remedy this situation and provide this explanation using Structured Flows on Manifolds (SFMs) (Jirsa, 2020; Pillai and Jirsa, 2017; Huys et al., 2014; McIntosh and Jirsa, 2019; Woodman and Jirsa, 2013). SFMs is a mathematical framework explaining how low dimensional dynamics, reflecting generative sets of rules underlying behavior, emerges in high-dimensional nonlinear systems, specifically dynamical systems on networks modeling macroscale brain dynamics. When properly linked to the network's constituent entities (functional nodes and connectivity), we will demonstrate how their causal activities lead to the formation of brain's resting SFM, comprising all its dynamic signatures (see Figure 1). If we distill the previous reports of brain resting state data analysis from the dynamical systems point of view, we arrive at the following main empirical signatures that should be part of the end stage of a successful mechanistic description: bistability of single region activation (Watanabe et al., 2013, 2014; Gu et al., 2018), low-dimensionality of the global system dynamics in state space (Deco et al., 2011; Ashourvan et al., 2017; Vohryzek et al., 2020), cascade propagation (Rabuffo et al., 2021), multistability of recurrent coactivation spatial patterns (beim Graben et al., 2019; Cornblath et al., 2020) and their non-trivial temporal dynamics or intermittency (Wong and Wang, 2006; Hansen et al., 2015; Esfahlani et al., 2020). These signatures will constitute the key features of what we will describe as structured flows on the low dimensional resting state manifold. 


\section{Disconnected}

\section{Broken symmetry}

\section{Fully connected}
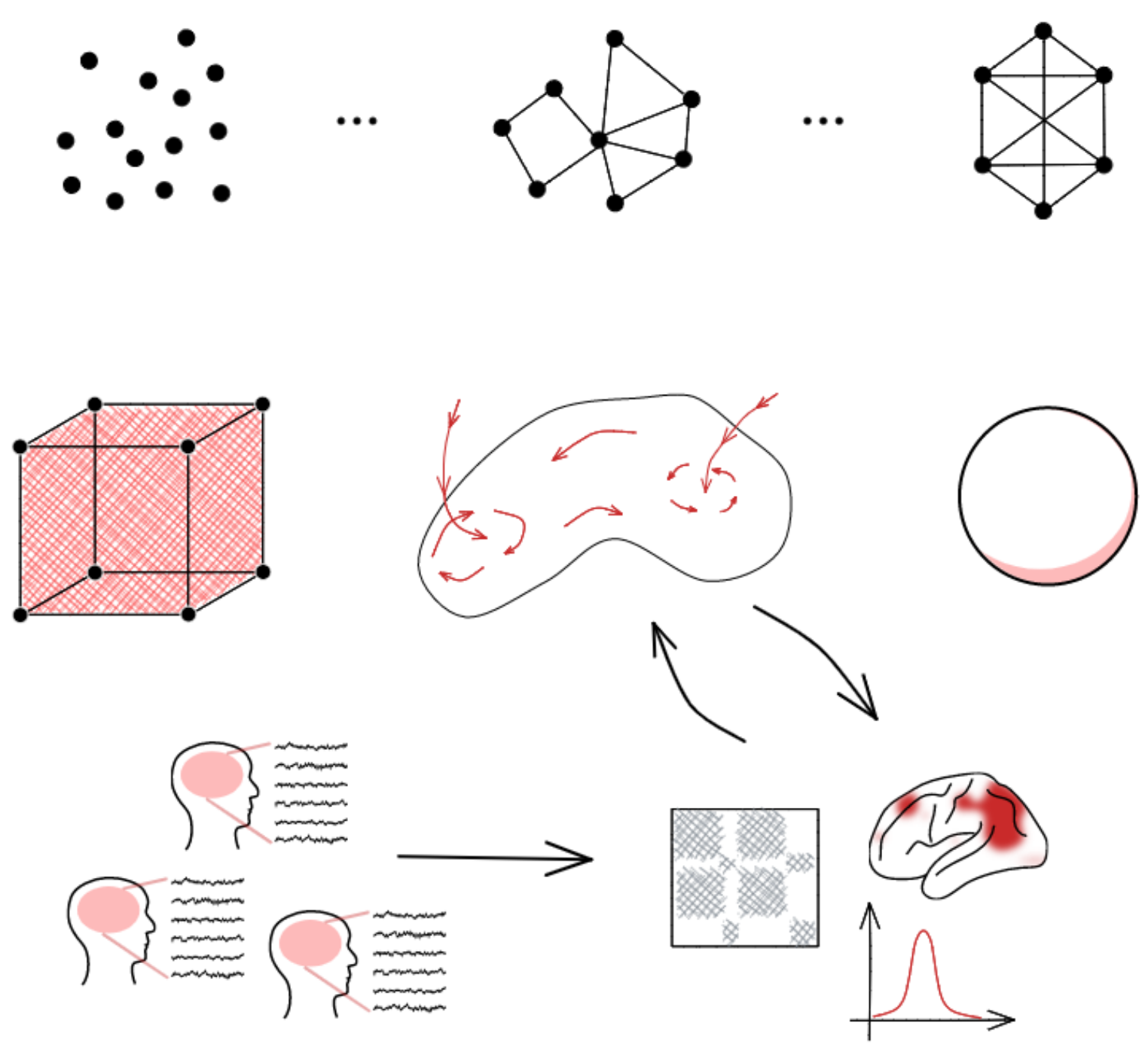

Figure 1. Structured flows on manifolds as focus of resting state characterization. With respect to the structure of the connectivity of the dynamical system, we consider spectrum defined by the two symmetrical limit cases: fully connected and fully disconnected network. Driven by noise, the disconnected system exhibits fully statistical, high-dimensional dynamics - it explores the whole state space in a equidirectional manner. On the other hand, the dynamics of the fully connected system is fully constrained corresponding to a $\mathrm{SO}(\mathrm{n})$ hypersphere with zero flow. The dynamics on the sparsely connected system leads to an object in between - a low-dimensional attractive manifold with an associated flow (SFM). It is this object we wish to put in the center of interest and characterize. While the SFM object remains the same, connections are made to data of various modalities with the help of suitable data features.

\section{Results}

In what follows we employ whole-brain modeling to study the low dimensional manifold and the associated structured flows of the spontaneous resting state dynamics, and how these relate to the structural connectome. We constructed a whole brain network model in the Virtual Brain (Sanz-Leon et al., 2013) using the two-dimensional neural mass model of quadratic integrate-and-fire neurons (Montbrió et al. (2015); MPR) to govern the regional dynamics coupled with a 
connectome derived from a subject from the Human Connectome Project (Van Essen et al., 2013). We have applied Balloon-Windkessel model (Stephan et al., 2007) to the simulated neuronal mass activity to generate realistic BOLD signals. We computed the Dynamical Functional Connectivity (dFC) from the BOLD signals to capture the changes in the system's dynamics on the slow time scale, and applied the Principal Component Analysis (PCA) to the model time series in the $2 \mathrm{~N}$-dimensional state space to capture the low-dimensional manifold on which the system evolves (see Materials and Methods for more details).

When driven by noise, the network of the bistable MPR nodes has the capacity to exhibit realistic dFC when the network input is scaled appropriately (Rabuffo et al., 2021). The noise together with the network input drives the switching between up- and down-state of the individual nodes, while the network mediates the coordination reflected in the functional connectivity. In the following sections, we explore how the manifold of the resting state activity arises from the networked interactions and how it shapes the multistability of the functional connectivity in the simulated BOLD.

\section{Symmetry breaking: working point for dFC}

To assess the impact of the symmetry breaking by the connectome, we simulated 10 minutes of spontaneous activity for a range of values of the coupling scaling parameter $G$ and noise variance $\sigma$, and applied PCA to the source signal $\Psi(t)$ and $\mathrm{dFC}$ to the BOLD (Figure 2). We used the variance accounted for (VAF) of the first two PCA components as an estimate for the dimensionality of the system's dynamics in the state-space (Figure 2D), and the variance of the upper triangle of the dFC matrix as a measure of the fluidity of the system's dynamics- that is the ability to dwell in specific brain states (defined by the functional connectivity) and shift between several such states (Figure 2A). We have observed, that for low values of $G$, the system exhibits high-dimensional dynamics as reflected in the low variance explained by the first PCA components and low values of the variance of the off-diagonal values of dFC with the mean around 0 -reflecting the absence of recurrence in the system dynamics (Figure 2B,C). Around the value of $G=G_{w}=0.525$ and $\sigma_{w}=0.030$ (working point) the variance explained by the first two components of the PCA increases substantially, and so does the fluidity of the dFC as the characteristic intervals of FC invariance (on-diagonal nonzero blocks) appear together with similarity across time (high off-diagonal correlations). Past the working point $(G>0.6)$ the explained variance in PCA drops as well as the off-diagonal dFC correlations, signifying increase in dimensionality of the spontaneous dynamics. 


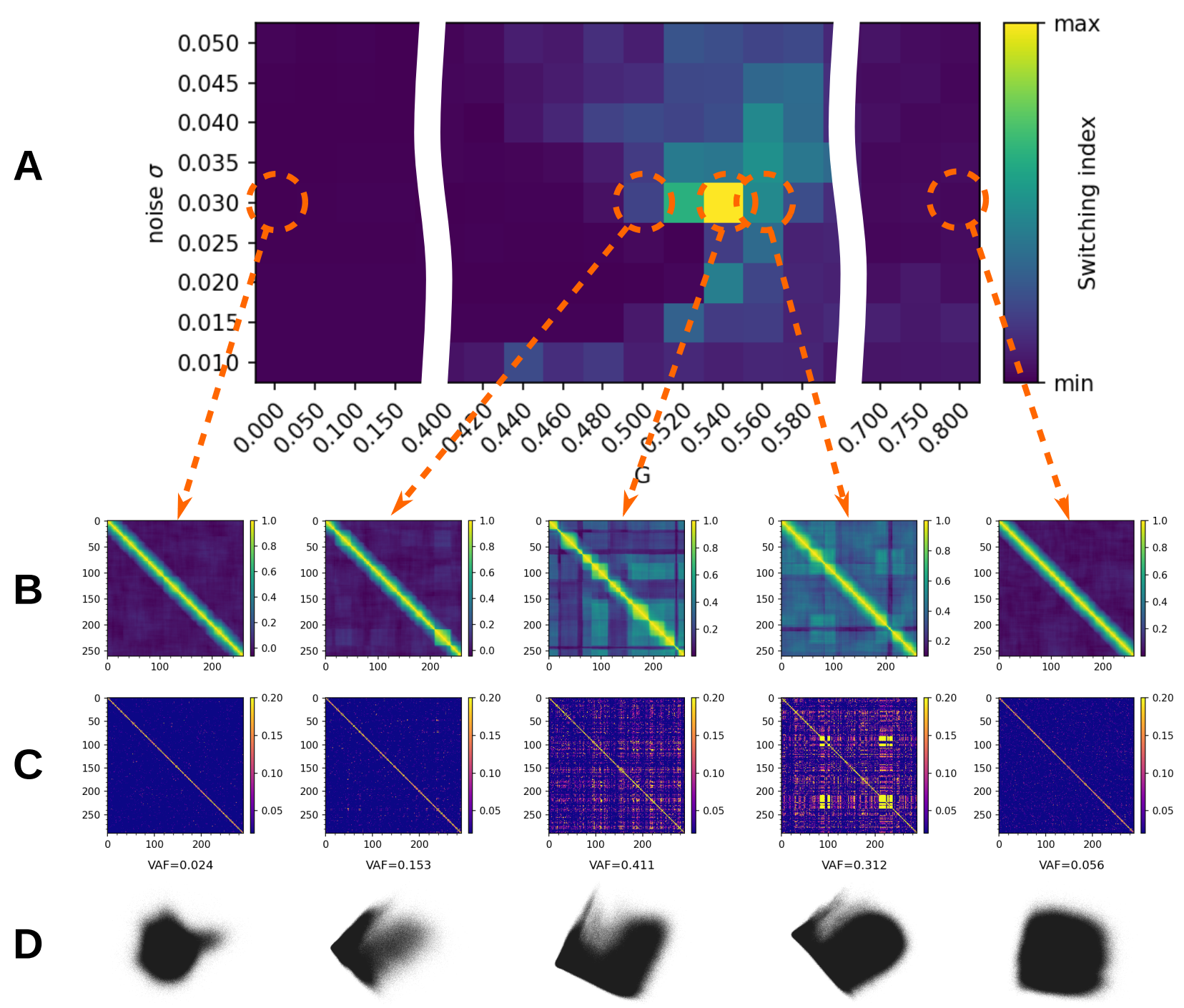

Figure 2. Brain network model and symmetry breaking. The brain network model is simulated for varying levels of global coupling parameter $G$ and noise variance $\sigma$ to produce both time-series of the state space variables $\mathbf{r}(t), \mathbf{V}(t)$, and the BOLD signal. For each combination of $G$ and $\sigma$ we compute the sliding window $d F C_{w}$ matrix from the simulated BOLD signal, and quantify the "switching index" of the dFC as the variance of the upper triangle (A). For selected values of $(G, \sigma)$ we show the sliding window $d F C_{w}(\mathrm{~B})$, edge based $d F C_{e}(\mathrm{C})$ and the projection of $\mathbf{r}(t)$ time series in the first two PCA components (D) annotated with corresponding fractional variance accounted for (VAF). In the working point around $G=0.54$ and intermediate values of $\sigma$ the system exhibits recurrence in the large-scale dynamics as captured by non-zero switching index, and reduction of dimensionality as captured in the increase in explained variance by the first PCA components and the asymmetry in the respective projection. For values of $G$ below or above the working point, the systems loses the fluidity property as reflected in the absence of the off-diagonal blocks on the dFC, and exhibits high-dimensional dynamics.

\section{Network dynamics}

Before we delve into the characterization of the low-dimensional manifold, let us first describe the network dynamics in detail. For the MPR model, the dynamical profile of an isolated node in the bistable parametrization consists of an unstable fixed point (saddle node) and two stable fixed points: down-state stable node and up-state focus (Figure 3A). Considering the uncoupled system, that is, the joint dynamics of the $\mathrm{N}$ populations (nodes) in the absence of any 
inter-population synaptic coupling, the phase flow is dominated by $2^{N}$ stable fixed points that represent all possible combinations of the populations firing at either their low or high mean firing rates (down or up state, respectively). Starting from an initial condition, the system will settle into the nearest accessible such fixed point, a stable network state composed of a corresponding combination of regions in their up or down state. Thus, the dynamics of the uncoupled system in phase space can be thought of as being driven by a potential energy landscape with multiple stable local minima representing the stable attractor states of the network. In fact, the system, as such, is invariant under permutation of the indexes of the populations, such that these latter attractor network states are distinguished only in terms of the respective number of nodes in up and down states. The global dynamics of the system, thus, collapses in finite time onto this stable attractor composed of a finite set of stable equilibrium points that is invariant under shuffling of indexes of the nodes. The associated global phase flow can be decomposed into $\mathrm{N}$ projections onto the identical 2D phase planes of individual populations, depicted in Figure 3A. Viewed from this perspective, the structure of the basins of attraction of the $2^{N}$ stable system equilibrium points redundantly inherits, in higher dimensions, the relative structure of the basins of attraction of the two stable fixed points of an individual population. For an isolated node, varying the external input $I_{e}$ changes the size of the basins of attraction of the stable fixed points. This modulates the probability of switching between the two states when driven by noise as captured by the mean escape times (Figure 3A, see Methods for more detail). For connected node, the external input $I_{e}$ depends on the state of the neighboring nodes (see Equation 4), fluctuating as they transition between the up- and down-state. On the network level, given right scaling of the network connections, this enables the cascades of up- and down-state switching at the fast time-scale, and the co-fluctuation of the BOLD signal (Figure 3B).

To understand better the dynamical underpinning of the increase of fluidity of the dFC we assess the characteristics of the co-fluctuations of the BOLD signal and the cascades in the source signal. For the co-activations, we start from the edge time series which is defined as pairwise dot product of z-scored BOLD signal (an average over the edge time series would correspond to the pearson correlation). The correlation across time-points yield the $d F C_{e}$ matrix capturing the recurrence of the edge configurations, and the root sum squared (RSS) over the edges at each time point captures the contribution of that particular time point to the overall functional connectivity (see Methods for more details). The time-points crossing the 95-th percentile threshold of the RSS are considered as strong co-activation events. The neuronal cascades (Rabuffo et al., 2021) are long lasting perturbations of the neuroelectric activity and are measured on a global level as a sum over regions of the binarized firing rate activity (at the threshold of 3 standard deviations). These measures we compared between the working point $G_{w}$, the disconnected system $G=0$, the strong network coupling regime $G>>G_{w}$, and the empirical data (Figure 3C,D).

In the working point $G_{w}$ the co-activations include large number of edges (Figure 3D) and the RSS follows the number of cascades up to a short delay corresponding to the delay of the BOLD signal. Moreover, the some of the strong co-activations re-occur partially in time as reflected in the non-zero elements of the $d F C_{e}$ matrix. Same characteristics can be observed in the empirical data, namely in the simultaneous EEG and fMRI measurements. On the other hand, the characteristic spatial and temporal structure is lost outside of the working point, that is either for the weakly coupled system $\left(G<<G_{w}\right)$, or for too strong coupling $\left(G>>G_{w}\right)$.

To quantify how the co-activation events contribute to the characteristic similarity across time, we compare the correlation of the edge vectors during the events, during the non-events, and between events and non-events. As a result we observe increased similarity of the edge vectors during the events both in the empirical data and in the simulations in the working point $G_{w}$. Again, this property is lost for weak $\left(G<<G_{w}\right)$ or for too strong coupling $\left(G>>G_{w}\right)$. Together, these results show, that the system has a similar dynamical profile in the working point $G_{w}$ as observed in the empirical data with respect to the network-carried fluctuations on both the fast and slow timescales (as captured by $d F C$ and cascades respectively). 
A

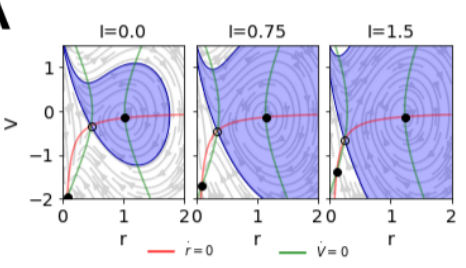

C

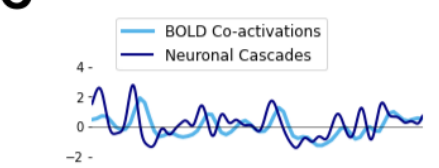

D
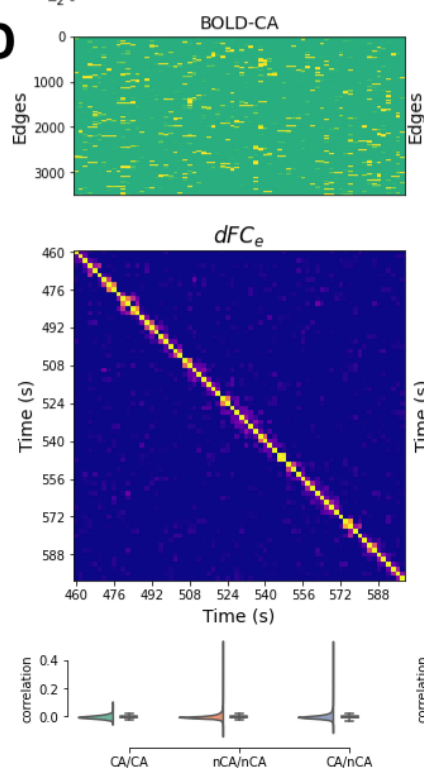

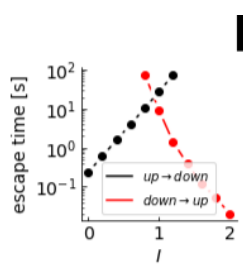

B

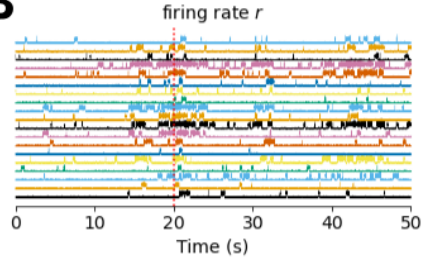

$\mathrm{G}=0.54$
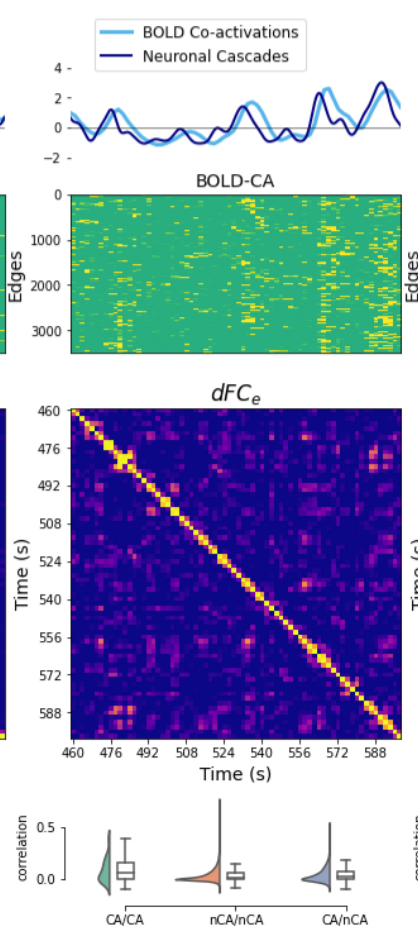

$\mathrm{G}=1.0$

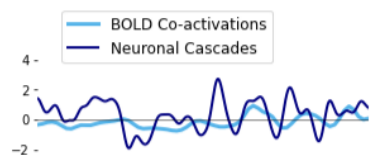

BOLD-CA

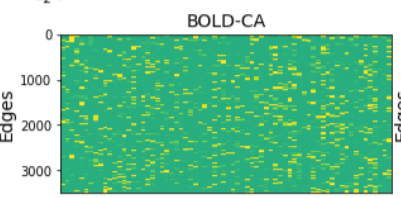

$d F C_{e}$
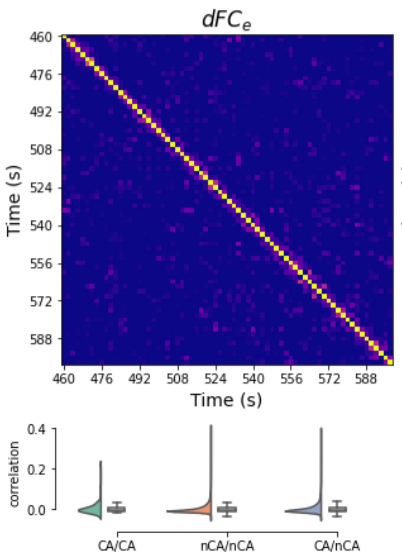

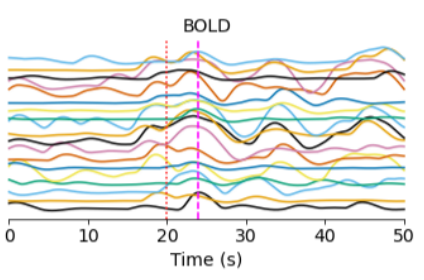

empirical

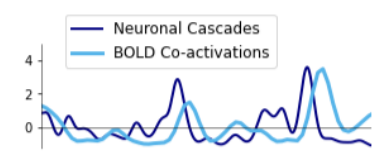

BOLD-CA

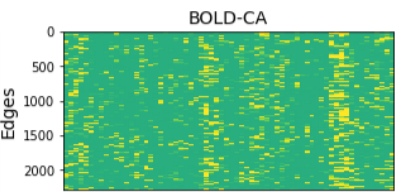

$d F C_{e}$
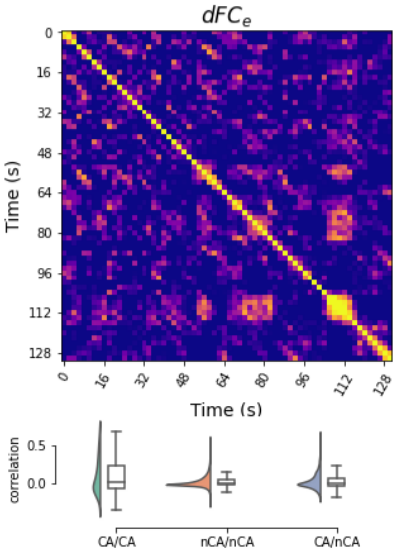

Figure 3. Network dynamics. (A) The network input $I$ modulates the probability of a noise-driven transition between the down- and up-state. (B) Example of a cascade - coordinated increase in activity translating to a delayed correlated peak in BOLD signal. Below we compare the network dynamics in and outside the working point, and the empirical data. In both empirical data and the working point $(G=0.54)$, the BOLD co-activations follow the neuronal cascades with a delay $(C)$, and show distinct spatial profiles which are recurrent in time (D).

\section{Manifold of the resting state and characteristic subspaces}

Having characterized the dynamics of the system in the working point with an appropriate measure, let us now relate these to the trajectories of the system in the $2 \mathrm{~N}$-dimensional state space. To do this, we first select time intervals with similar functional connectivity. Starting from the edge time series, we have clustered the time points using k-means $(\mathrm{k}=5)$, which separated the high-activity intervals (majority of the nodes in the up-state), low-activity intervals (majority of the nodes in the down-state), and the co-fluctuation events (Figure 4A).

Next, we identified the trajectories of the system underlying each cluster in the low dimensional projection of state space. For each cluster, we have selected the corresponding time points in the state space of the system, and projected them into the first two principal components of the PCA computed on the complete time series. We have observed that while the corresponding subspaces overlap partially in the projection (Figure 4B), the activity within the clusters concentrates to different subspaces. This concentration in different subspaces is reflected in the distance between the centroids of the cluster time points in the PCA projection.

While the cluster activity overlaps in the projection in the components of the PCA computed from the whole time 
series, the co-activation trajectories can be separated by choosing different basis to span the low-dimensional space, that is compute the PCA from the time points corresponding to the co-activation clusters. To project the trajectories observed at the slow time scale of the BOLD on the manifold, we have shifted the BOLD signal by the characteristic lag, and for each BOLD time point belonging to the cluster selected the corresponding time points in $\mathbf{r}(t)$ convolved with Gaussian kernel of similar width as the width of the BOLD response function (see Methods for details). As the number of time points included in the three co-fluctuation clusters is small (by definition), we have merged them for this step into a single category. We have then spanned the subspace corresponding to the first two PCA components of the co-fluctuation trajectories, and projected the remaining two clusters into this space. This procedure have separated the non-events trajectories from the events trajectories (Figure 4C).

To get a more precise information on the occurrence of both the co-fluctuations and cascades on the manifold, we have computed the binned averages of the RSS and the cascade participation in the space of the first two PCA components of the $\mathbf{r}(t)$ time series. Both the cascades and high-amplitude co-fluctuations concentrate in this projection at the outer part of the high-activity subspace (Figure 4D). Furthermore, to describe the flow on the manifold, we computed the average transition direction from each bin in the same projection as before. We found, that the flow at the boundary of the projection directs the trajectories towards the inside, where it doesn't have a structure in the low-activity subspace, and where it is strongly directional within the subspace of co-fluctuations and cascades-pointing back towards the high-activity subspace. Taken together, these results chart the low-dimensional manifold of the system in the working point regime, associating the subspaces with specific flows. The fluid dynamics as characterized in previous section then arise from the slow transitions between the low- and high-activity subspace, where the latter supports the strong co-activation events which are reflected in the $d F C$. 
A

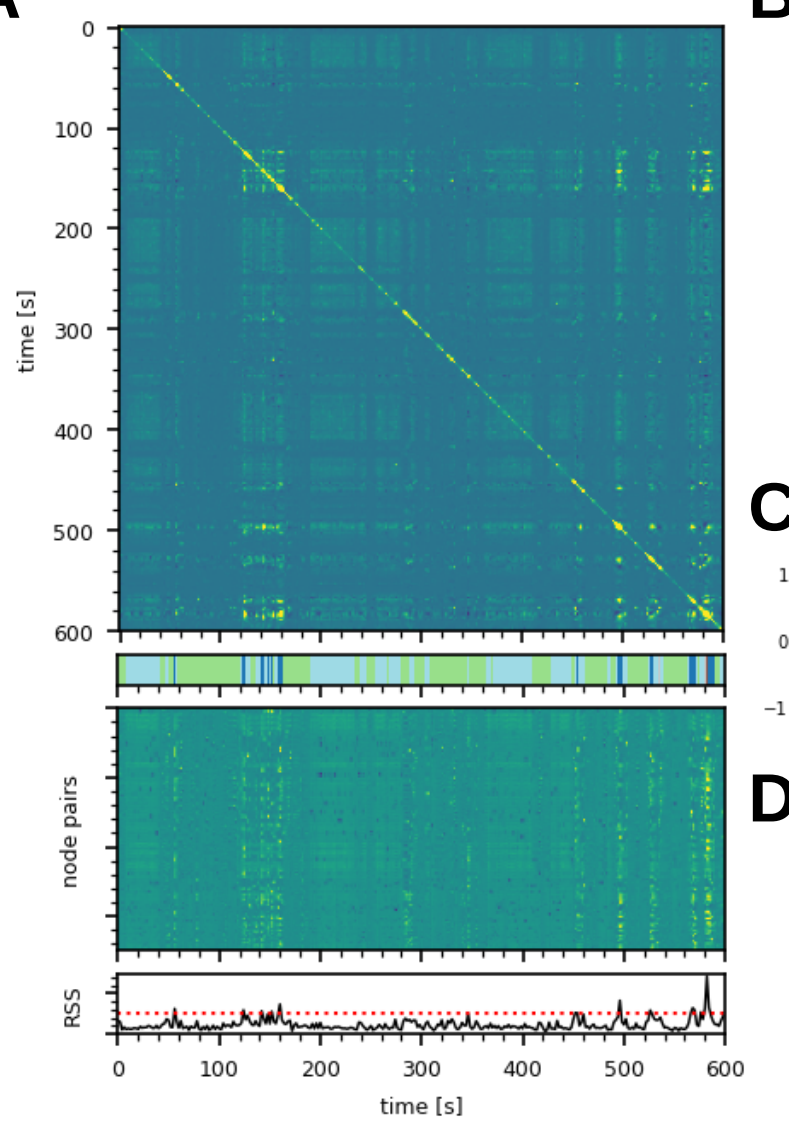

B
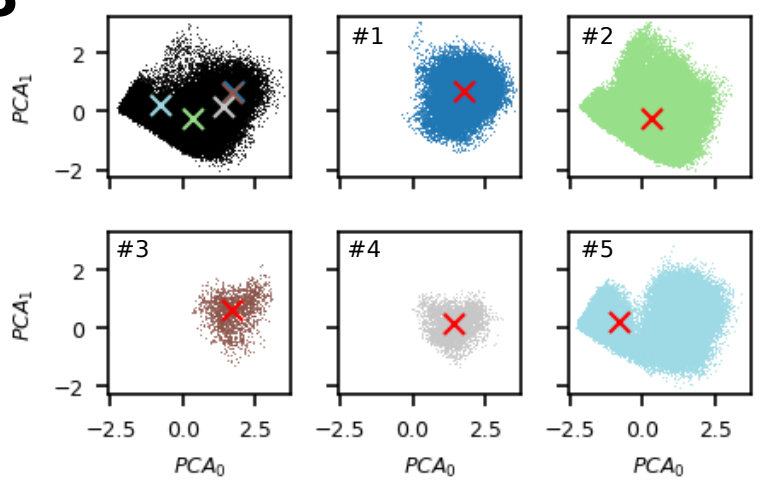

clusters $1,3,4$

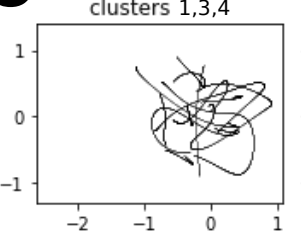

cluster 2
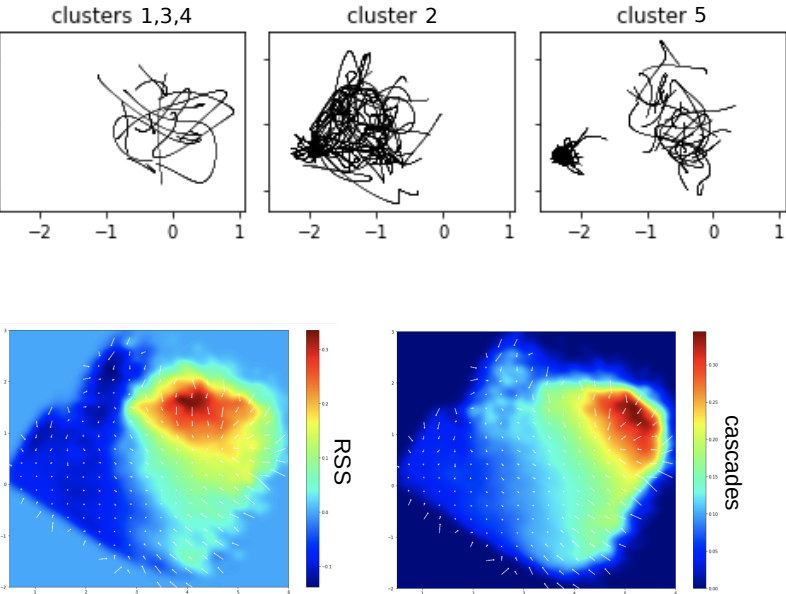

Figure 4. Manifold subspaces and characteristic dynamics. (A) The edge time series shows characteristic co-activation events defined as time points with the root sum squared (RSS) in the 95th percentile, which are similar across time as reflected in the covariance matrix of the edge time series (top). (B) The edge time series was divided into 5 clusters (k-means) and the corresponding time intervals in $\mathbf{r}(t)$ were selected; in the first panel the centroids of the time points are shown in the projection to the first two principal components, following panels show the projection of the $\mathbf{r}(t)$ intervals of particular clusters. Cluster \# 2 captures the high-activity subspace, cluster \#5 corresponds to the low-activity state, and the clusters \#1, \#3, and \#4 capture the co-activation events. (C) Local trajectories of the manifold subspaces: 0-delay BOLD surrogate time series with fine time resolution was partitioned according the 5 subspaces, and projected to the space defined by first two components of the PCA applied to the time series of the clusters \#1, \#3, and \#4. (D) The average flow (white arrows) and the heat map of number of cascades (right) and co-fluctuation strength (left).

\section{Fixed point skeleton and structured flow}

To understand how the resting state manifold arises, we start by considering the uncoupled system, that is, the joint dynamics of the $N$ populations (nodes) in the absence of any iter-population synaptic coupling. This uncoupled system's phase flow is dominated by $2^{N}$ stable fixed points that represent all possible combinations of the populations firing at either their low or high mean firing rates (down or up state, respectively). Starting from an initial condition and in the absence of noise, the system will settle into the nearest accessible such fixed point, a stable network state composed of a corresponding combination of regions in their up or down state.

The dynamical effects of the permutation symmetry breaking are delineated by the topology of the connectome. The heterogeneity of the in-degree (total connectivity) of individual nodes of the network drives a variation in the relative positioning of the separatrices between the basins of attraction of the equilibrium points, mirrored in the variation of the corresponding projections onto the 2D phase planes of corresponding nodes (see Figure 3A). In conjunction, 
connectivity strength and topology give rise to gradients in the relative attractiveness of the system's equilibrium states, reflected in variations of the largest negative eigenvalues obtained from the linearization of the system about the respective equilibrium state.

To map the complete manifold outside the simulated trajectories we sampled the stable fixed points for varying coupling scaling parameter $G$ from the $2^{N}$ combinations of up- and down-states, and evaluated their stability (see Methods for more details). We found that the number of stable fixed points in the sample decreases with increasing $G$. This decrease is due to the loss of states with mixed composition of up- and down-state due to the bifurcation of the down state in nodes with high input (Figure 5A). Projecting the $\mathbf{r}$ component of the fixed-points in the first two eigenvectors of the Laplacian confirms this thinning of the intermediate compositions biased towards those with higher number of nodes in the up-state (corresponds to the first Laplacian eigenvector $\lambda_{0}$ ). Additionally, the stability of the fixed points was inversely proportional to the number of nodes in the up-state, that is in the direction of $E_{0}$ the first eigenvector of the Laplacian (Figure 5B).

To put this into the context of the simulated trajectories, we have next identified the fixed points around whose the simulated trajectory evolved by taking initial conditions from the simulated trajectory, and integrating the system without noise to the equilibrium. We have confirmed that in all instances the system reached a stable fixed point composed of combination of up- and down-states, and that the stability of these fixed points follows the same gradient in terms of the composition (Figure 5C).

Furthermore, the nodes of the network exhibit a frequency gradient of the oscillations in the up-state (Figure 5D). This gradient reflects variability of the characteristic frequency in the up-state across nodes in the network. In the fixed-point state, if the nodes are treated as isolated systems with an input current term based on the existing network state, then

$$
\begin{aligned}
& r_{i}^{*}=r^{*}+\delta_{i}^{r} \\
& v_{i}^{*}=v^{*}+\delta_{i}^{v}
\end{aligned}
$$

where $\left(r^{*}, v^{*}\right)$ are the symmetric fixed-points of the network and $\left(\delta_{i}^{r}, \delta_{i}^{v}\right)$ are the excursions from the symmetric fixedpoint and change according to the existing network state. These excursions depend directly on the in-strength of the $i$ th node and the local states of its first neighbours.

Following linear stability analysis of the $i$ th system around the fixed-point (see Methods), the eigenvalues of the Jacobian matrix are given by

$$
\begin{aligned}
\lambda_{1,2} & =2 v_{i}^{*} \pm \sqrt{J r_{i}^{*}-4 \pi^{2} r_{i}^{*^{2}}} \\
& =2 v_{i}^{*} \pm \sqrt{2 J r_{i}^{*}-4 \pi 2 r_{i}^{*^{2}}+2 J \delta_{i}^{r}-4 \pi^{2} \delta_{i}^{r^{2}}-8 \pi^{2} r^{*} \delta_{i}^{r}}
\end{aligned}
$$

From the above equation, we see that the frequency of oscillations in the up-state of the $i$ th node increases proportional to $\delta_{i}^{r}$ and therefore proportional to the in-strength of the node, which we also observe in the simulation (Figure 5E).

Finally, we have compared the gradient of the dominant frequency peak to the empirical values obtained from intracranial recordings (Wang et al., 2021). We have observed, that the model captures the general front-to-back trend on the level of brain lobes, that is a decrease of the frequency from frontal to parietal, temporal and to occipital lobe (Figure 5I). 

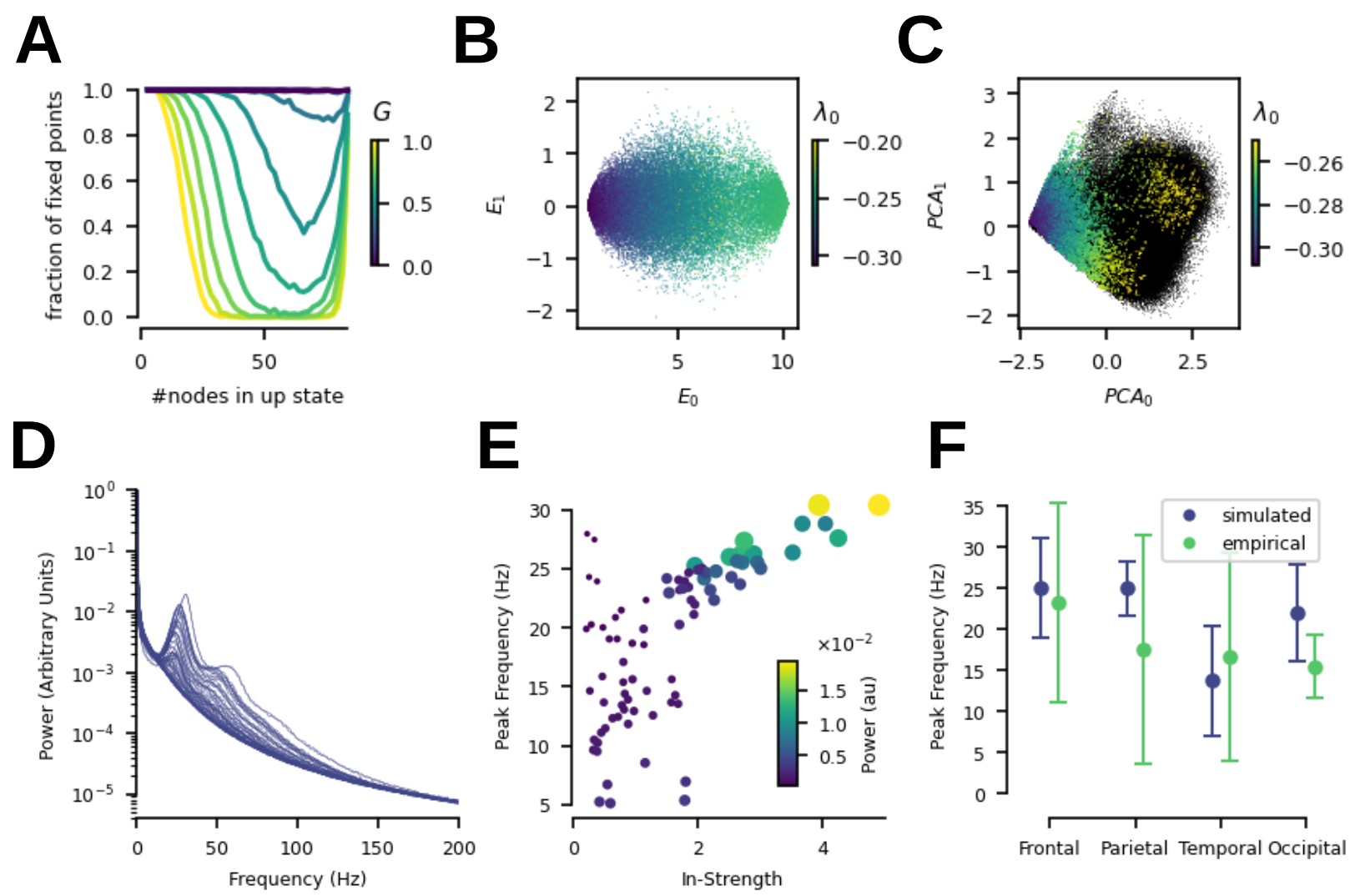

Figure 5. Mechanistic structure of the manifold. (A) composition of the sampled stable fixed points in terms of number of nodes in the up-state as a function of $G$, normalized to $G=0$. (B) projection of the stable fixed points into the first two leading eigenmodes of the network Laplacian $E_{0}, E_{1}$, color codes the value of the largest eigenvalue in the linear stability analysis. (C) fixed points (colored) derived by noise-free integration to equilibrium from the trace of a simulation (black) in the working point $G_{w}$, color codes the value of the largest eigenvalue $\lambda_{0}$ in the linear stability analysis. From the simulated source activity, we extract the power-spectrum for each brain-region (D). From these spectra, the peak-frequency is identified for each region. In (E), we plot the peak-frequency against the in-strength of each region and color indicating the corresponding power. Consistent with the earlier reasoning, we observe a linear trend, especially for frequencies with higher power. The average peak-frequency, weighted by the respective power, is calculated for the frontal, parietal, temporal and occipital lobes. This frequency gradient across the lobes is compared to the gradient obtained from empirical data $(\mathrm{F})$.

\section{Discussion}

Using a combination of computational modeling and dynamical systems analysis we have provided a complete mechanistic description in terms of constituent entities and their causal activities leading to spontaneous co-activations and neuronal cascades in the brain's resting state (Rabuffo et al., 2021). We showed how the breaking of the symmetry of the system's connectivity gives rise to the structured low-dimensional dynamics in the phase space and recurrent fluctuations of the functional connectivity (Figure 2). These fluctuations arise from network-mediated cascades of upand down-state switching and capture well the empirically found relationship between the strong co-activation events and the recurrence structure reflected by the functional connectivity dynamics (Figure 3). The subspace accessible to the system in this regime was charted and partitioned according to the characteristic flow associated with each of the partition (Figure 4). Finally, this subspace and its associated flows arise from the rich fixed point structure of the system and the differential stability of the nodes in these fixed points is not only reflected in the propensity to state switching, but also influences the dominant oscillation frequency in the up-state (Figure 5). In summary, these results support our 
hypothesis that the recurrent functional connectivity states of the resting state correspond to distinct subspaces on a low-dimensional manifold associated with distinct structured flows.

The central result of our work is that the symmetry breaking via the structural connectivity carves out an attractive subspace of all the possible system's configurations, and that the flow on this manifold governs the characteristic dynamics of the system (that is discarding the transient towards the manifold from arbitrary initial conditions). In this regime, the model captures the multistability and noise-driven exploration of the dynamic repertoire explored previously in computational studies (Ghosh et al., 2008; Deco and Jirsa, 2012; Haimovici et al., 2013; Hansen et al., 2015; Courtiol et al., 2020). The data features extracted from the time series provide a surface of contact between the empirical data and the model. Here, the functional structure in the system is carried by the rare high amplitude co-fluctuation events as it was previously demonstrated in empirical fMRI data (Tagliazucchi et al., 2012; Esfahlani et al., 2020; Sporns et al., 2021), and in simultaneous EEG and fMRI measurements (Rabuffo et al., 2021). Similarly, recent modeling study has shown the role of structural modules of the network in shaping the co-fluctuation events (Pope et al., 2021), which is aligned with the network as the symmetry breaking feature of the system giving rise to the low-dimensional dynamics.

The slow time scale fluctuations of the dynamical functional connectivity reflect the movement of the system between the low- and high-activity subspaces of the manifold, where the flow in the high-activity subspace supports the cascades, which in turn are reflected in the high activity coactivations. This movement points to the multistable rather then metastable interpretation of the resting state dynamics (Cocchi et al., 2017), and reflects the observation of switching between a low-amplitude incoherent and high-amplitude coherent states in empirical data (Kong et al., 2021). Furthermore, the slow transitions between the high- and low-activity subspaces is compatible with the reports on the spontaneous infra-slow brain activity (Fox and Raichle, 2007; Mitra et al., 2018) and the detailed reports on its spatio-temporal structure. For example, the slow traveling waves ( $G \boldsymbol{u}$ et al., 2021) propagating along the principal gradient of cortical organization (Margulies et al., 2016; Huntenburg et al., 2018) would provide a refined description of the trajectory through the manifold subspaces.

The attractive subspace of the low-dimensional manifold and the associated structured flow arise in the presented system from the changes in the fixed-point structure due to the irregular connectivity. In particular, the network input mediates the modulation of the escape times of the noise-induced transitions, which chain into domino-like sequences (Ashwin et al., 2017, 2018) which in turn constitute the neuronal cascades. On a network level, our results elaborate the previous analytical results of increased entropy of the attractors in an Ising-spin network model for intermediate values of coupling strength (Deco et al., 2012). The relationship to the dimensionality of the exhibited dynamics is such that for the low values of coupling strength $G$, where the Ising model is in the trivial state with all spins equal to 0 , the model presented here is also driven by noise to the all-down state due to the significantly larger basin of attraction of the down-state, and the nodes make uncoordinated noise-driven excursions to the up-state reflected in the high-dimensionality of the dynamics. For high values of $G$ the situation is opposite, and for intermediate values of $G$ the Ising model exhibits high entropy of attractors, which is in our case reflected in the available states organized in the low-dimensional manifold with the structured flow governed by the stability of these states.

Overall, the movement of the system through the subspaces of a low-dimensional manifold is in accordance with empirical and modeling results on recurrence and state clustering of resting state fMRI BOLD time series. Using clustering algorithms to partition the BOLD time series yields statistically similar and temporally recurrent whole brain spatial coactivation patterns (beim Graben et al., 2019; Cornblath et al., 2020) associated with specific dwell times and transition probabilities. However, compared to the clustering approaches applied to the BOLD time-series, the SFMs allows us to refine the partitioning of the state-space in two aspects: we unfold the subspaces based on the similarity of the coactivations on the level of the BOLD signal, and provide a detailed description of the flow of the system in these subspaces e.g. in terms of the cascades. Interestingly, the clustering of phase-locking BOLD states (Vohryzek et al., 2020) leads to very similar low-dimensional representation of the resting state dynamics to our approach, with a single 
dominant global phase locked state and a number of transient partial phase-locked states related to functional networks. Similarly, embedding the resting state data onto the task manifold extracted with the help of diffusion maps (Gao et al., 2021) found the resting state time-points concentrate in the task-fixation and transition subspaces complemented by a minority of time points in the cognitive subspaces of the manifold.

The description of the structured flow addresses also the fast time-scale by including the cascades, which we previously showed to relate to the co-activations observed in the BOLD signal (Rabuffo et al., 2021). In EEG literature, the spatio-temporal structure of the resting state dynamics is characterized with the help of microstates-sensor-level transient patterns lasting on average for 60-150 ms (Michel and Koenig, 2018). Attempts have been made to relate the microstates to BOLD activation clusters (Britz et al., 2010; Bréchet et al., 2019), but identifying the sources generating the microstates with clustering or regression analysis has been challenging so far due to unclear relationship between the broadband EEG activity and the BOLD signal fluctuations. To advance we propose to reframe the question as a search for a shared manifold of the neuronal activity, which is reflected both in the EEG and the BOLD observables.

The manifold we describe is conceptually reminiscent of energy landscapes described in previous works (Watanabe et al., 2014; Gu et al., 2018). However, previous energy landscape models, such as in (Gu et al., 2018), implicitly assume energy minimization and thus, by construction, encode the hypothesis that the activation of two brain regions that are connected via a direct structural connection is more energetically favorable than that of two regions that are not directly connected. We make no such assumption here and, instead, the effective energy landscape emerges, in the form of a low dimensional manifold, out of the interplay of the non-linearity in the local neural mass model and the structural connectivity. In addition, previous energy landscape analysis (Watanabe et al., 2014) assumed that the network changes only gradually by flipping one region at a time, and did not account for transitions in which several regions flip simultaneously. The model we presented here instead allows for such latter transitions of the system in state space, which may very well be due to strongly connected regions that are able to simultaneously influence their nearest neighbors during coactivation events. An intriguing direction for the extension of the framework presented here is in more refined inclusion of the subcortical structures. Notably, recent works (Shine, 2020; Müller et al., 2020; Munn et al., 2021) exploring the role of thalamus, locus coeruleus, and basal nucleus of Meynert in shaping of the dynamical landscape of the cortical activity are already formulated in the dynamical systems language while incorporating carefully the detailed anatomical and cytoarchitectural knowledge. Integrating these advances in the SFM framework is a natural next step towards the original motivation of SFM, that is to link the mesoscopic neuronal activity to the behaviour, as the intricate interactions between cortex and the subcortical areas are one of the organizing principles of the underlying the biological mechanisms supporting behaviour (Cisek, 2019).

In conclusion, our results show how the low-dimensional dynamics arises from breaking the symmetry in the system on the level of the connectome. Describing these dynamics as structured flows on manifolds allows us to bridge the gap between the observational measures and the state-space trajectories of the system. As such, this object is well suited for comparison across different models, scales, and neuroimaging modalities, and provides means for integration of the diverse descriptions of the resting state dynamics.

\section{Materials and methods}

\section{Brain network model}

Computational brain network model (Sanz-Leon et al., 2015) is used to simulate resting state activity under varying values of network coupling scaling parameter $G$. The dynamics of each of the network nodes were governed by the neural mass model (NMM) derived analytically as the limit of infinitely all-to-all coupled $\theta$-neurons (Montbrió et al., 2015), namely for $i$-th node as:

$$
\begin{aligned}
\tau_{m} \dot{r}_{i} & =\frac{\Delta}{\pi \tau_{m}}+2 r_{i} v_{i}, \\
\tau_{m} \dot{v}_{i} & =v_{i}^{2}+\eta-\left(\tau_{m} \pi r_{i}\right)^{2}+J \tau_{m} r_{i}+I_{i} .
\end{aligned}
$$


The $N$ nodes are then coupled with a connectome derived from empirical data as

$$
I_{i}(t)=G \sum_{j} W_{i j} r_{j}\left(t-D_{i j}\right),
$$

where $G$ is the network scaling parameter, $W_{i j}$ is the connection weight $D_{i j}=L_{i j} / v$ is the delay caused by propagation of the signal on a tract of length $L_{i j}$ with finite velocity $v$. We picked the speed $v=2 \mathrm{~m} / \mathrm{s}$ from the biologically plausible range (Trebaul et al., 2018).

The equations 3 and 4 comprise the drift $a(\Psi, t)$ in the stochastic delay differential equation formulation with linear additive noise reading:

$$
d \Psi(t)=a(\Psi(t)) d t+b(\Psi(t)) d W(t)
$$

where $\Psi$ is the state vector $\left[\psi_{1}, \ldots \psi_{n}\right]$ with $\psi_{n}=\left[r_{n}, V_{n}\right], d W(t)$ is a differential of a Wiener process with Gaussian increment with variance $\sigma^{2}$, and $b(\Psi, t)=1$ is the diffusion coefficient—here constant yielding the noise term additive.

The model was implemented in The Virtual Brain (Sanz-Leon et al., 2013) and equipped with BOLD forward solution comprising the Balloon-Windkessel model applied to the firing rate $\mathbf{r}(t)$ (Stephan et al., 2007).

The model parameters $\eta=-5.0, J=15.0$, and $\Delta=1.0$ were selected to set the nodes in the bi-stable regime in the absence of coupling (Rabuffo et al., 2021). We then varied the global coupling parameter $G$ and the noise variance $\sigma$, and simulated the 10 minutes of resting state BOLD activity after discarding 10 seconds of the initial transient.

\section{Functional connectivity dynamics}

In order to track the time-dependent changes in the functional connectivity, we compute the windowed dynamical functional connectivity $d F C_{w}$ (Allen et al., 2014; Hansen et al., 2015) and $d F C_{e}$ (Rabuffo et al., 2021). Starting from the regional BOLD time-series $B_{n}(t)$ for each node $n$, we compute functional connectivity matrices $F C(w)$ for each time window $w=1 \ldots W$ defined as $\left.B_{n}(t)\right|_{t_{w}} ^{t_{w}+\tau}$ with window length $\tau=60 \mathrm{~s}$ and window step size $t_{(w+1)}-t_{w}=2 s$. Next we compute the $d F C_{w}$ matrix of order $W$ as

$$
d F C_{w}(i, j)=\operatorname{corr}\left(F C\left(w_{i}\right)^{\triangle}, F C\left(w_{j}\right) \triangle\right),
$$

where $F C(w)^{\triangle}$ is the vectorized upper part of the $F C$ matrix.

For the window-less $d F C_{e}$ (Rabuffo et al., 2021) we start from the edge time-series (Esfahlani et al., 2020) defined as $E_{n m}(t)=z_{n}(t) z_{m}(t)$ for $n, m=1 \ldots N$ where $z_{n}(t)=\frac{B_{n}-\mu_{n}}{\sigma_{n}}$ is the z-scored BOLD time-series of a node $n$. The edge dynamical functional connectivity is then computed as correlation between the edge vectors at each pair of time points $t_{1}, t_{2}$ :

$$
d F C_{e}\left(t_{1}, t_{2}\right)=\operatorname{corr}\left(E_{n m}\left(t_{1}\right), E_{n m}\left(t_{2}\right)\right) .
$$

The co-fluctuation events (CF) are defined as time points in the edge time-series $E_{n m}(t)$ during which the root sum squared $R S S=\sqrt{\sum_{n m} E_{n m}^{2}(t)}$ crosses a given threshold, here chosen as 95th percentile. Time points where $R S S$ is below the threshold are then labeled as non-events (nCF).

The avalanches were computed on the binary mask $\mathbf{a}(t)$ on the $\mathbf{r}(t)$ such that $a_{i}(t)=1 \Longleftrightarrow z\left(r_{i}(t)\right)>3$ where $z\left(r_{i}(t)\right.$ is the $\mathrm{z}$-score of firing rate $r$ of a node $i$.

\section{Manifold subspaces}

As a first step in the analysis of the local dynamics specific to a particular attractive subspace, we have extracted the time-points belonging to these subspaces with k-means clustering applied to the edge time series $E_{n m}(t)$. We varied the number of clusters $k$ and selected $k=5$ at which the co-fluctuation events separated to distinct cluster.

To extract the segments of $\mathbf{r}(t)$ corresponding to the $E_{n m}(t)$ time points we first estimated the BOLD signal lag $l=2500 \mathrm{~ms}$ as optimal peak-to-peak alignment with $\mathbf{r}(t)$ smoothened by a Gaussian filter with same effective width 
$(\sigma=700)$. Then for all BOLD time points in a given cluster $c$ we selected the 2000 corresponding time points in $\mathbf{r}(t)$ and concatenate these to get the subspace $\mathbf{r}_{c}(t)$.

\section{Manifold sampling}

To identify the fixed point scaffold of the manifold as traced by the trajectory resulting from integrating the Equation 5, we sample the segments from the simulated trajectory $\left.\left(r_{i}(t), v_{i}(t)\right)\right|_{t_{s}} ^{t_{s}+\tau_{\max }}$, and use them as initial conditions for integration of the deterministic interpretation of Equation 5, i.e. $d \Psi(t) \stackrel{s}{=} a(\Psi(t)) d t$. From each such an initial condition, we integrated the system to steady state equilibrium corresponding to a fixed point $\left(\mathbf{r}^{*}, \mathbf{v}^{*}\right)$.

The number of stable fixed points $\left(\mathbf{r}^{*}, \mathbf{v}^{*}\right)$ of system with $G=0$ is $2^{N}$ reflecting all the combinations of up- and down-states of the $N$ nodes. To sample the stable fixed points of the system with $G>0$ we solve repeatedly the system of equations:

$$
\begin{aligned}
& 0=\frac{\Delta}{\pi \tau_{m}}+2 r_{i}^{*} v_{i}^{*}, \\
& 0=v_{i}^{* 2}+\eta-\left(\tau_{m} \pi r_{i}^{*}\right)^{2}+J \tau_{m} r_{i}^{*}+I_{i}
\end{aligned}
$$

using Newton-Raphson method with the initial conditions chosen randomly as a vector of up- and down-state fixed points of the isolated nodes, i.e. $\left(r_{i}^{* 0}, v_{i}^{* 0}\right) \in\left\{\left(r_{\uparrow}^{*}, v_{\uparrow}^{*}\right),\left(r_{\downarrow}^{*}, v_{\downarrow}^{*}\right)\right\}, \forall i$ where $\left(r_{\uparrow}^{*}, v_{\uparrow}^{*}\right)$ and $\left(r_{\downarrow}^{*}, v_{\downarrow}^{*}\right)$ are the up- and down-state fixed points for the isolated node. For each initial condition $\left(\mathbf{r}^{* 0}, \mathbf{v}^{* 0}\right)$ we then check if the corresponding solution of Equation 8 is equivalent up to the composition in terms of up- and down-states. If not, it is discarded, otherwise we evaluate the stability of the found fixed point using linear stability analysis.

As a low-dimensional projection of the sampled manifold we have used the two slowest eigenmodes of the structural connectivity. These are computed as eigendecomposition of the graph Laplacian $\mathbf{L}=\mathbf{W}-\mathbf{I}$, that is $\mathbf{L U}=\mathbf{U} \mathbf{\Lambda}$, where eigenvalues $\lambda_{k}$ can be interpreted as structural freqencies and the eigenmodes $\mathbf{u}_{k}$ as structural connectome harmonics (Preti and Van De Ville, 2019).

\section{Linear stability analysis}

We perform a linear stability analysis to identify the fixed-points obtained from the NR method. If each fixed-point $\left(\mathbf{r}^{*}, \mathbf{v}^{*}\right)$ is perturbed by $\left(\epsilon^{\mathbf{r}}, \epsilon^{\mathbf{v}}\right)$, then the evolution of the perturbations depend on the Jacobian matrix $(J)$ and are given by:

$$
\left[\begin{array}{c}
\dot{\epsilon}_{1}^{r} \\
\dot{\epsilon}_{2}^{r} \\
\vdots \\
\dot{\epsilon}_{N}^{r} \\
\dot{\epsilon}_{1}^{v} \\
\dot{\epsilon}_{2}^{v} \\
\vdots \\
\dot{\epsilon}_{N}^{v}
\end{array}\right]=\left[\begin{array}{cccccccc}
2 v_{1}^{*} & 0 & \ldots & 0 & 2 r_{1}^{*} & 0 & \ldots & 0 \\
0 & 2 v_{2}^{*} & \ldots & 0 & 0 & 2 r_{2}^{*} & \ldots & 0 \\
\vdots & \vdots & \ddots & \vdots & \vdots & \vdots & \ddots & \vdots \\
0 & 0 & \ldots & 2 v_{N}^{*} & 0 & 0 & \ldots & 2 v_{N}^{*} \\
J-2 \pi^{2} r_{1}^{*} & w_{12} & \ldots & w_{1 N} & 2 v_{1}^{*} & 0 & \ldots & 0 \\
w_{21} & J-2 \pi^{2} r_{2}^{*} & \ldots & w_{2 N} & 0 & 2 v_{2}^{*} & \ldots & 0 \\
\vdots & \vdots & \ddots & \vdots & \vdots & \vdots & \ddots & \vdots \\
w_{N 1} & w_{N 2} & \ldots & J-2 \pi^{2} r_{N}^{*} & 0 & 0 & \ldots & 2 v_{N}^{*}
\end{array}\right] \cdot\left[\begin{array}{c}
\epsilon_{1}^{r} \\
\epsilon_{2}^{r} \\
\vdots \\
\epsilon_{N}^{r} \\
\epsilon_{1}^{v} \\
\epsilon_{2}^{v} \\
\vdots \\
\epsilon_{N}^{v}
\end{array}\right]
$$

The stability of a fixed-point depends on the eigenvalues of the Jacobian evaluated at the fixed-point. The fixed-point is stable iff all the eigen-values of $\boldsymbol{J}$ are negative. Therefore, we numerically evaluate the largest eigenvalue of Jacobian for each fixed-point and label the point as stable if its real-part is negative.

\section{Fixed point sampling from simulated trajectory}

From a given trajectory of the system given as 10 minutes of $\psi(t)$ we have selected a restart point $t^{\prime}$ each $50 \mathrm{~ms}$ (12000 starting points altogether). For each of the restart point $t^{\prime}$ we extracted the segment $\left.\Psi(t)\right|_{t^{\prime}} ^{t^{\prime}-\tau_{\max }}$ where $\tau_{\max }$ is the length 
of the longest delay, and used as initial condition to a equivalent system to Equation 5 with $b=0$ :

$$
d \Psi(t)=a(\Psi(t)) d t .
$$

Integrating this system to equilibrium yielded then for each restart point $t_{r}$ a fixed point $\Psi^{*}=\left(\mathbf{r}^{*}, \mathbf{v}^{*}\right)$. The stability of each of the fixed points $\Psi^{*}$ was then evaluated using the linear stability analysis as the largest eigenvalue of the respective Jacobian matrix.

\section{Escape time analysis}

The switching behaviour of a single node is driven by the stability of the up- and down-state fixed points in the presence of noise. We employed escape time analysis (Yamapi et al., 2010) to measure the stability of these fixed points for range of values of external input $I$. In detail, for a single node of the system given by Equation 3 we found the up- and down-state stable fixed points $\left(r^{*}, v^{*}\right)^{\uparrow}$ and $\left(r^{*}, v^{*}\right)^{\downarrow}$, and the unstable saddle node $\left(r^{*}, v^{*}\right)^{\times}$. Next we computed the separatrix between the two basins of attraction by integration of the model backwards in time resulting in an closed curve $\omega$. To find the characteristic escape time for a fixed point $\left(r^{*}, v^{*}\right)$ we have integrated the system from the initial condition $\left(r_{0}, v_{0}\right)=\left(r^{*}, v^{*}\right)$ for a given value of $I 100$ times, measuring the time $t_{E}$ at which the trajectory crosses $\omega$ for the first time. The values of $I$ were drawn from the range given by $\left[0, I_{\max }\right]$ where $I_{\max }=\max \left\{I_{i}(t), \forall i\right\}$ is the largest value of $I_{i}$ encountered in the integration of the full system in the working point.

\section{References}

Allen EA, Damaraju E, Plis SM, Erhardt EB, Eichele T, Calhoun VD. Tracking whole-brain connectivity dynamics in the resting state. Cerebral Cortex. 2014 Mar; 24(3):663-676. doi: https://doi.org/10.1093/cercor/bhs352.

Ashourvan A, Gu S, Mattar MG, Vettel JM, Bassett DS. The energy landscape underpinning module dynamics in the human brain connectome. Neuroimage. 2017; 157:364-380. doi: https://doi.org/10.1016/j.neuroimage.2017.05.067.

Ashwin P, Creaser J, Tsaneva-Atanasova K. Fast and slow domino regimes in transient network dynamics. Phys Rev E. 2017 Nov; 96(5-1):052309. doi: https://doi.org/10.1103/PhysRevE.96.052309.

Ashwin P, Creaser J, Tsaneva-Atanasova K. Sequential escapes: onset of slow domino regime via a saddle connection. Eur Phys J Spec Top. 2018 Nov; 227(10):1091-1100. doi: https://doi.org/10.1140/epjst/e2018-800038-5.

Baker AP, Brookes MJ, Rezek IA, Smith SM, Behrens T, Probert Smith PJ, Woolrich M. Fast transient networks in spontaneous human brain activity. Elife. 2014 Mar; 3:e01867. doi: https://doi.org/10.7554/eLife.01867.

Battaglia D, Boudou T, Hansen ECA, Lombardo D, Chettouf S, Daffertshofer A, McIntosh AR, Zimmermann J, Ritter P, Jirsa V. Dynamic Functional Connectivity between order and randomness and its evolution across the human adult lifespan. Neuroimage. 2020 Nov; 222:117156. doi: https://doi.org/10.1016/j.neuroimage.2020.117156.

Braun U, Schaefer A, Betzel RF, Tost H, Meyer-Lindenberg A, Bassett DS. From Maps to Multi-dimensional Network Mechanisms of Mental Disorders. Neuron. 2018 Jan; 97(1):14-31. doi: https://doi.org/10.1016/j.neuron.2017.11.007.

Breakspear M. Dynamic models of large-scale brain activity. Nat Neurosci. 2017 Feb; 20(3):340-352. doi: https://doi.org/10.1038/nn.4497.

Bréchet L, Brunet D, Birot G, Gruetter R, Michel CM, Jorge J. Capturing the spatiotemporal dynamics of self-generated, task-initiated thoughts with EEG and fMRI. Neuroimage. 2019 Jul; 194:82-92. doi: https://doi.org/10.1016/j.neuroimage.2019.03.029.

Britz J, Van De Ville D, Michel CM. BOLD correlates of EEG topography reveal rapid resting-state network dynamics. Neuroimage. 2010; 52(4):1162-1170. doi: https://doi.org/10.1016/j.neuroimage.2010.02.052.

Cabral J, Kringelbach ML, Deco G. Functional connectivity dynamically evolves on multiple time-scales over a static structural connectome: Models and mechanisms. Neuroimage. 2017 Oct; 160:84-96. doi: https://doi.org/10.1016/j.neuroimage.2017.03.045. 
Cavanna F, Vilas MG, Palmucci M, Tagliazucchi E. Dynamic functional connectivity and brain metastability during altered states of consciousness. Neuroimage. 2018 Oct; 180(Pt B):383-395. doi: https://doi.org/10.1016/j.neuroimage.2017.09.065.

Cisek P. Resynthesizing behavior through phylogenetic refinement. Atten Percept Psychophys. 2019 Oct; 81(7):2265-2287. doi: https://doi.org/10.3758/s13414-019-01760-1.

Cocchi L, Gollo LL, Zalesky A, Breakspear M. Criticality in the brain: A synthesis of neurobiology, models and cognition. Prog Neurobiol. 2017 Nov; 158:132-152. doi: https://doi.org/10.1016/j.pneurobio.2017.07.002.

Cornblath EJ, Ashourvan A, Kim JZ, Betzel RF, Ciric R, Adebimpe A, Baum GL, He X, Ruparel K, Moore TM, Gur RC, Gur RE, Shinohara RT, Roalf DR, Satterthwaite TD, Bassett DS. Temporal sequences of brain activity at rest are constrained by white matter structure and modulated by cognitive demands. Commun Biol. 2020 May; 3(1):261. doi: https://doi.org/10.1038/s42003020-0961-x.

Courtiol J, Guye M, Bartolomei F, Petkoski S, Jirsa VK. Dynamical mechanisms of interictal resting-state functional connectivity in epilepsy. Journal of Neuroscience. 2020; 40(29):5572-5588. doi: 10.1523/JNEUROSCI.0905-19.2020.

Damoiseaux JS, Rombouts SARB, Barkhof F, Scheltens P, Stam CJ, Smith SM, Beckmann CF. Consistent resting-state networks across healthy subjects. Proc Natl Acad Sci U S A. 2006 Sep; 103(37):13848-13853. doi: https://doi.org/10.1073/pnas.0601417103.

Deco G, Jirsa V, McIntosh AR, Sporns O, Kötter R. Key role of coupling, delay, and noise in resting brain fluctuations. Proc Natl Acad Sci U S A. 2009 Jun; 106(25):10302-10307. doi: https://doi.org/10.1073/pnas.0901831106.

Deco G, Jirsa VK. Ongoing cortical activity at rest: criticality, multistability, and ghost attractors. J Neurosci. 2012 Mar; 32(10):33663375. doi: https://doi.org/10.1523/JNEUROSCI.2523-11.2012.

Deco G, Jirsa VK, McIntosh AR. Emerging concepts for the dynamical organization of resting-state activity in the brain. Nat Rev Neurosci. 2011 Jan; 12(1):43-56. doi: https://doi.org/10.1038/nrn2961.

Deco G, Jirsa VK, McIntosh AR. Resting brains never rest: computational insights into potential cognitive architectures. Trends Neurosci. 2013 May; 36(5):268-274. doi: https://doi.org/10.1016/j.tins.2013.03.001.

Deco G, Kringelbach ML, Arnatkeviciute A, Oldham S, Sabaroedin K, Rogasch NC, Aquino KM, Fornito A. Dynamical consequences of regional heterogeneity in the brain's transcriptional landscape. Sci Adv. 2021 Jul; 7(29):eabf4752. doi: https://doi.org/10.1126/sciadv.abf4752.

Deco G, Senden M, Jirsa V. How anatomy shapes dynamics: a semi-analytical study of the brain at rest by a simple spin model. Front Comput Neurosci. 2012 Sep; 6:68. doi: https://doi.org/10.3389/fncom.2012.00068.

Esfahlani FZ, Jo Y, Faskowitz J, Byrge L, Kennedy DP, Sporns O, Betzel RF. High-amplitude cofluctuations in cortical activity drive functional connectivity. Proc Natl Acad Sci U S A. 2020 Nov; 117(45):28393-28401. doi: https://doi.org/10.1073/pnas.2005531117.

Fox MD, Raichle ME. Spontaneous fluctuations in brain activity observed with functional magnetic resonance imaging. Nat Rev Neurosci. 2007 Sep; 8(9):700-711. doi: https://doi.org/10.1038/nrn2201.

Gao S, Mishne G, Scheinost D. Nonlinear manifold learning in functional magnetic resonance imaging uncovers a low-dimensional space of brain dynamics. Hum Brain Mapp. 2021 Oct; 42(14):4510-4524. doi: https://doi.org/10.1002/hbm.25561.

Ghosh A, Rho Y, McIntosh AR, Kötter R, Jirsa VK. Noise during rest enables the exploration of the brain's dynamic repertoire. PLoS Comput Biol. 2008 Oct; 4(10):e1000196. doi: https://doi.org/10.1371/journal.pcbi.1000196.

beim Graben P, Jimenez-Marin A, Diez I, Cortes JM, Desroches M, Rodrigues S. Metastable Resting State Brain Dynamics. Frontiers in Computational Neuroscience. 2019; 13. doi: https://doi.org/10.3389/fncom.2019.00062.

Gu S, Cieslak M, Baird B, Muldoon SF, Grafton ST, Pasqualetti F, Bassett DS. The Energy Landscape of Neurophysiological Activity Implicit in Brain Network Structure. Sci Rep. 2018 Feb; 8(1):2507. doi: https://doi.org/10.1038/s41598-018-20123-8. 
Gu Y, Sainburg LE, Kuang S, Han F, Williams JW, Liu Y, Zhang N, Zhang X, Leopold DA, Liu X. Brain Activity Fluctuations Propagate as Waves Traversing the Cortical Hierarchy. Cereb Cortex. 2021 Jul; 31(9):3986-4005. doi: https://doi.org/10.1093/cercor/bhab064.

Gusnard DA, Raichle ME, Raichle ME. Searching for a baseline: functional imaging and the resting human brain. Nat Rev Neurosci. 2001 Oct; 2(10):685-694. doi: https://doi.org/10.1038/35094500.

Haimovici A, Tagliazucchi E, Balenzuela P, Chialvo DR. Brain organization into resting state networks emerges at criticality on a model of the human connectome. Phys Rev Lett. 2013 Apr; 110(17):178101. doi: https://doi.org/10.1103/PhysRevLett.110.178101.

Hansen ECA, Battaglia D, Spiegler A, Deco G, Jirsa VK. Functional connectivity dynamics: modeling the switching behavior of the resting state. Neuroimage. 2015 Jan; 105:525-535. doi: https://doi.org/10.1016/j.neuroimage.2014.11.001.

Honey CJ, Kötter R, Breakspear M, Sporns O. Network structure of cerebral cortex shapes functional connectivity on multiple time scales. Proc Natl Acad Sci U S A. 2007 Jun; 104(24):10240-10245. doi: https://doi.org/10.1073/pnas.0701519104.

Huntenburg JM, Bazin PL, Margulies DS. Large-Scale Gradients in Human Cortical Organization. Trends Cogn Sci. 2018 Jan; 22(1):21-31. doi: https://doi.org/10.1016/j.tics.2017.11.002.

Hutchison RM, Womelsdorf T, Allen EA, Bandettini PA, Calhoun VD, Corbetta M, Della Penna S, Duyn JH, Glover GH, Gonzalez-Castillo J, Handwerker DA, Keilholz S, Kiviniemi V, Leopold DA, de Pasquale F, Sporns O, Walter M, Chang C. Dynamic functional connectivity: promise, issues, and interpretations. Neuroimage. 2013 Oct; 80:360-378. doi: https://doi.org/10.1016/j.neuroimage.2013.05.079.

Huys R, Perdikis D, Jirsa VK. Functional architectures and structured flows on manifolds: a dynamical framework for motor behavior. Psychol Rev. 2014 Jul; 121(3):302-336. doi: https://doi.org/10.1037/a0037014.

Jirsa V. Structured Flows on Manifolds as guiding concepts in brain science. In: Selbstorganisation-ein Paradigma für die Humanwissenschaften Springer; 2020.p. 89-102. doi: https://doi.org/10.1007/978-3-658-29906-4_6.

Jones DT, Vemuri P, Murphy MC, Gunter JL, Senjem ML, Machulda MM, Przybelski SA, Gregg BE, Kantarci K, Knopman DS, Boeve BF, Petersen RC, Jack CR Jr. Non-stationarity in the "resting brain's" modular architecture. PLoS One. 2012 Jun; 7(6):e39731. doi: https://doi.org/10.1371/journal.pone.0039731.

Kong X, Kong R, Orban C, Wang P, Zhang S, Anderson K, Holmes A, Murray JD, Deco G, van den Heuvel M, Yeo BTT. Sensory-motor cortices shape functional connectivity dynamics in the human brain. Nat Commun. 2021 Nov; 12(1):6373. doi: https://doi.org/10.1038/s41467-021-26704-y.

Liu X, Zhang N, Chang C, Duyn JH. Co-activation patterns in resting-state fMRI signals. Neuroimage. 2018 Oct; 180 (Pt B):485-494. doi: https://doi.org/10.1016/j.neuroimage.2018.01.041.

Machamer P, Darden L, Craver CF. Thinking about mechanisms. Philosophy of science. 2000; 67(1):1-25.

Margulies DS, Ghosh SS, Goulas A, Falkiewicz M, Huntenburg JM, Langs G, Bezgin G, Eickhoff SB, Castellanos FX, Petrides M, Jefferies E, Smallwood J. Situating the default-mode network along a principal gradient of macroscale cortical organization. Proc Natl Acad Sci U S A. 2016 Nov; 113(44):12574-12579. doi: https://doi.org/10.1073/pnas.1608282113.

McIntosh AR, Jirsa VK. The hidden repertoire of brain dynamics and dysfunction. Netw Neurosci. 2019 Sep; 3(4):994-1008. doi: https://doi.org/10.1162/netn\_a\_00107.

Melozzi F, Bergmann E, Harris JA, Kahn I, Jirsa V, Bernard C. Individual structural features constrain the mouse functional connectome. Proc Natl Acad Sci U S A. 2019 Dec; doi: https://doi.org/10.1073/pnas.1906694116.

Michel CM, Koenig T. EEG microstates as a tool for studying the temporal dynamics of whole-brain neuronal networks: A review. Neuroimage. 2018 Oct; 180(Pt B):577-593. doi: https://doi.org/10.1016/j.neuroimage.2017.11.062. 
Mišić B, Betzel RF, de Reus MA, van den Heuvel MP, Berman MG, McIntosh AR, Sporns O. Network-Level Structure-Function Relationships in Human Neocortex. Cereb Cortex. 2016 Jul; 26(7):3285-3296. doi: https://doi.org/10.1093/cercor/bhw089.

Mitra A, Kraft A, Wright P, Acland B, Snyder AZ, Rosenthal Z, Czerniewski L, Bauer A, Snyder L, Culver J, Lee JM, Raichle ME. Spontaneous Infra-slow Brain Activity Has Unique Spatiotemporal Dynamics and Laminar Structure. Neuron. 2018 Apr; 98(2):297-305.e6. doi: https://doi.org/10.1016/j.neuron.2018.03.015.

Montbrió E, Pazó D, Roxin A. Macroscopic description for networks of spiking neurons. Physical Review X. 2015; 5(2):021028. doi: https://doi.org/10.1103/physrevx.5.021028.

Müller EJ, Munn BR, Shine JM. Diffuse neural coupling mediates complex network dynamics through the formation of quasi-critical brain states. Nat Commun. 2020 Dec; 11(1). doi: https://doi.org/10.1038/s41467-020-19716-7.

Munn BR, Müller EJ, Wainstein G, Shine JM. The ascending arousal system shapes neural dynamics to mediate awareness of cognitive states. Nat Commun. 2021 Oct; 12(1):6016. doi: https://doi.org/10.1038/s41467-021-26268-x.

Pillai AS, Jirsa VK. Symmetry Breaking in Space-Time Hierarchies Shapes Brain Dynamics and Behavior. Neuron. 2017 jun; 94(5):1010-1026. doi: https://doi.org/10.1016/j.neuron.2017.05.013.

Pope M, Fukushima M, Betzel RF, Sporns O. Modular origins of high-amplitude cofluctuations in fine-scale functional connectivity dynamics. Proc Natl Acad Sci U S A. 2021 Nov; 118(46). doi: https://doi.org/10.1073/pnas.2109380118.

Preti MG, Bolton TA, Van De Ville D. The dynamic functional connectome: State-of-the-art and perspectives. Neuroimage. 2017 Oct; 160:41-54. doi: https://doi.org/10.1016/j.neuroimage.2016.12.061.

Preti MG, Van De Ville D. Decoupling of brain function from structure reveals regional behavioral specialization in humans. Nat Commun. 2019 Oct; 10(1):4747. doi: https://doi.org/10.1038/s41467-019-12765-7.

Rabuffo G, Fousek J, Bernard C, Jirsa V. Neuronal Cascades Shape Whole-Brain Functional Dynamics at Rest. eNeuro. 2021 Sep; 8(5). doi: https://doi.org/10.1523/ENEURO.0283-21.2021.

Roberts JA, Gollo LL, Abeysuriya RG, Roberts G, Mitchell PB, Woolrich MW, Breakspear M. Metastable brain waves. Nat Commun. 2019 Mar; 10(1):1056. doi: https://doi.org/10.1038/s41467-019-08999-0.

Sanz-Leon P, Knock SA, Spiegler A, Jirsa VK. Mathematical framework for large-scale brain network modeling in The Virtual Brain. NeuroImage. 2015; 111:385-430. doi: https://doi.org/10.1016/j.neuroimage.2015.01.002.

Sanz-Leon P, Knock SA, Woodman MM, Domide L, Mersmann J, McIntosh AR, Jirsa V. The Virtual Brain: a simulator of primate brain network dynamics. Frontiers in neuroinformatics. 2013; 7. doi: https://doi.org/10.3389/fninf.2013.00010.

Shine JM, Breakspear M, Bell PT, Ehgoetz Martens K, Shine R, Koyejo O, Sporns O, Poldrack RA. The dynamic basis of cognition: an integrative core under the control of the ascending neuromodulatory system; 2018, doi: https://doi.org/10.1101/266635.

Shine JM, The thalamus integrates the macrosystems of the brain to facilitate complex, adaptive brain network dynamics. Elsevier Ltd; 2020. doi: https://doi.org/10.1016/j.pneurobio.2020.101951.

Shine JM, Bissett PG, Bell PT, Koyejo O, Balsters JH, Gorgolewski KJ, Moodie CA, Poldrack RA. The Dynamics of Functional Brain Networks: Integrated Network States during Cognitive Task Performance. Neuron. 2016 Oct; 92(2):544-554. doi: https://doi.org/10.1016/j.neuron.2016.09.018.

Sporns O, Faskowitz J, Teixeira AS, Cutts SA, Betzel RF. Dynamic expression of brain functional systems disclosed by fine-scale analysis of edge time series. Netw Neurosci. 2021 Apr; 5(2):405-433. doi: https://doi.org/10.1162/netn\_ą_00182.

Stephan KE, Weiskopf N, Drysdale PM, Robinson PA, Friston KJ. Comparing hemodynamic models with DCM. Neuroimage. 2007 Nov; 38(3):387-401. doi: https://doi.org/10.1016/j.neuroimage.2007.07.040. 
Tagliazucchi E, Balenzuela P, Fraiman D, Chialvo DR. Criticality in large-scale brain FMRI dynamics unveiled by a novel point process analysis. Front Physiol. 2012 Feb; 3:15. doi: https://doi.org/10.3389/fphys.2012.00015.

Trebaul L, Deman P, Tuyisenge V, Jedynak M, Hugues E, Rudrauf D, Bhattacharjee M, Tadel F, Chanteloup-Foret B, Saubat C, Reyes Mejia GC, Adam C, Nica A, Pail M, Dubeau F, Rheims S, Trébuchon A, Wang H, Liu S, Blauwblomme T, et al. Probabilistic functional tractography of the human cortex revisited. Neuroimage. 2018 Nov; 181:414-429. doi: https://doi.org/10.1016/j.neuroimage.2018.07.039.

Van Essen DC, Smith SM, Barch DM, Behrens TE, Yacoub E, Ugurbil K, Consortium WMH, et al. The WU-Minn human connectome project: an overview. Neuroimage. 2013; 80:62-79.

Vohryzek J, Deco G, Cessac B, Kringelbach ML, Cabral J. Ghost Attractors in Spontaneous Brain Activity: Recurrent Excursions Into Functionally-Relevant BOLD Phase-Locking States. Front Syst Neurosci. 2020 Apr; 14:20. doi: https://doi.org/10.3389/fnsys.2020.00020.

Wang HE, Scholly J, Triebkorn P, Sip V, Villalon SM, Woodman MM, Le Troter A, Guye M, Bartolomei F, Jirsa V. VEP atlas: An anatomic and functional human brain atlas dedicated to epilepsy patients. Journal of neuroscience methods. 2021; $348: 108983$. doi: https://doi.org/10.1016/j.jneumeth.2020.108983.

Watanabe T, Hirose S, Wada H, Imai Y, Machida T, Shirouzu I, Konishi S, Miyashita Y, Masuda N. A pairwise maximum entropy model accurately describes resting-state human brain networks. Nature communications. 2013; 4(1):1-10. doi: https://doi.org/10.1038/ncomms2388.

Watanabe T, Hirose S, Wada H, Imai Y, Machida T, Shirouzu I, Konishi S, Miyashita Y, Masuda N. Energy landscapes of resting-state brain networks. Front Neuroinform. 2014 Feb; 8:12. doi: https://doi.org/10.3389/fninf.2014.00012.

Wong KF, Wang XJ. A recurrent network mechanism of time integration in perceptual decisions. Journal of Neuroscience. 2006; 26(4):1314-1328. doi: https://doi.org/10.1523/jneurosci.3733-05.2006.

Woodman MM, Jirsa VK. Emergent dynamics from spiking neuron networks through symmetry breaking of connectivity. PloS one. 2013; 8(5):e64339.

Yamapi R, Filatrella G, Aziz-Alaoui MA. Global stability analysis of birhythmicity in a self-sustained oscillator. Chaos. 2010 Mar; 20(1):013114. doi: https://doi.org/10.1063/1.3309014.

Zalesky A, Fornito A, Cocchi L, Gollo LL, Breakspear M. Time-resolved resting-state brain networks. Proc Natl Acad Sci U S A. 2014 Jul; 111(28):10341-10346. doi: https://doi.org/10.1073/pnas.1400181111. 